\title{
ARTICLE
}

\section{Downregulation of parvalbumin expression in the prefrontal cortex during adolescence causes enduring prefrontal disinhibition in adulthood}

\author{
Adriana Caballero ${ }^{1}{ }^{1}$, Eden Flores-Barrera ${ }^{1}$, Daniel R. Thomases ${ }^{1}$ and Kuei Y. Tseng ${ }^{1}$
}

The expression of the calcium binding protein parvalbumin (PV) has been observed in several cortical regions during development in a temporal pattern consistent with increased afferent-dependent activity. In the prefrontal cortex (PFC), PV expression appears last and continues to substantially increase throughout adolescence, yet the significance of this increase remains unclear. Because of the expression of PV in fast-spiking GABAergic interneurons, we hypothesized that PV upregulation during adolescence is necessary to sustain the increase in GABAergic activity observed in the PFC during this period. To test this hypothesis, we utilized an RNAi strategy to directly downregulate PV levels in the PFC during adolescence and examined its impact on prefrontal GABAergic function, plasticity, and associated behaviors during adulthood. The data indicate that a mere $25 \%$ reduction of adult PV levels in the PFC was sufficient to reduce local GABAergic transmission onto pyramidal neurons, disrupt prefrontal excitatory-inhibitory balance, and alter processing of afferent information from the ventral hippocampus. Accordingly, these animals displayed an impairment in the level of extinction learning of a trace fear conditioning response, a behavioral paradigm that requires intact PFCventral hippocampus connectivity. These results indicate the PV upregulation observed in the PFC during adolescence is necessary for refinement of prefrontal GABAergic function, the absence of which results in immature afferent processing and a hypofunctional state. Importantly, these results suggest there is a critical window of plasticity during which PV upregulation supports the acquisition of mature GABAergic phenotype necessary to sustain adult PFC functions.

Neuropsychopharmacology (2020) 45:1527-1535; https://doi.org/10.1038/s41386-020-0709-9

\section{INTRODUCTION}

The elucidation of mechanisms mediating the transition from childhood to adulthood has received increasing attention under the premise that understanding cortical developmental trajectories will shed light onto disturbances leading to the onset of psychiatric disorders later in life [1]. As the cradle of cognitive functions, the prefrontal cortex (PFC) is one of the cortical regions whose development extends into adolescence [2, 3], a period during which neurotransmitter systems in the PFC undergo intense reconfiguration (reviewed in [4]). The PFC's involvement in developmental psychiatric disorders with cognition and affect dysregulation $[5,6]$ suggests its protracted maturation also extends its window of susceptibility until young adulthood. Nonetheless, the mechanisms conferring PFC vulnerability during adolescence remain largely undefined.

Parvalbumin (PV) is a member of the EF-hand family of calcium binding proteins with broad expression in the brain and muscle $[7,8]$. Long considered a "slow calcium buffer", the presence of PV grants the cells the ability to regulate the decay phase of the calcium transient [9-11], especially upon highfrequency stimulation [12]. In general, PV levels are strongly regulated throughout development [13-21], with mRNA and protein expression displaying an exquisite activity-dependence in both neural and nonneural tissue [22-26]. In the cortex, PV has been typically considered a marker for a subset of local
GABAergic interneurons which provide strong feedforward inhibition to pyramidal neurons [27]. In this role, PV-positive interneurons are considered major regulators of the excitatory-inhibitory balance in cortical circuits [28] and ultimately contribute to the organization and synchronization of afferent input [29]. Of relevance, the appearance of PV has been highly correlated with increased afferent drive into sensory cortices [23-25, 30-32]. Similarly, an increase of excitatory synaptic activity onto PV-positive interneurons occurs in parallel with a marked upregulation of PV protein expression in the PFC during adolescence [33].

The full picture of PV function in the nervous system is only beginning to be understood. The available data indicate that PV contributes to neurotransmitter release, broadly through regulation of calcium dynamics at the presynaptic site [11]. If true, the degree of PV expression could have a significant impact in all processes ascribed to PV-positive interneurons. This becomes especially important in the context of psychiatric disorders, particularly in schizophrenia where a reduction in PV expression has been found (see [34]). Of note, one report shows an approximate $20 \%$ reduction in PV mRNA expression without a loss in the number of PV-positive interneurons in the PFC of patients with schizophrenia [35]. Collectively, these results suggest that there is a threshold level of PV expression required to sustain adult PV-interneuron function below which deficits in cortical

${ }^{1}$ Department of Anatomy and Cell Biology, College of Medicine, University of Illinois at Chicago, Chicago, IL 60612, USA

Correspondence: Kuei Y. Tseng (tsengky@uic.edu)

Received: 21 February 2020 Revised: 4 May 2020 Accepted: 8 May 2020

Published online: 13 May 2020 
processing may occur. Here, we directly tested the hypothesis that peri-adolescent upregulation of PV in the PFC is necessary to sustain local inhibitory transmission and support normal prefrontal functions in adults.

\section{MATERIALS AND METHODS}

All experimental procedures were conducted according to the Guide for the Care and Use of Laboratory Animals, and approved by the UIC Animal Care Committee. Male Sprague Dawley rats (Envigo, IN) were group-housed (3 rats/cage), maintained at a constant temperature $\left(21-23^{\circ} \mathrm{C}\right)$, humidity, and light-dark cycle, and allowed to acclimate to the facility for at least 1 week before survival surgeries. Food and water were available ad libitum. See Supplemental Information for detailed methods.

Delivery of shRNA into the PFC

Commercially available rat PV- and scrambled-shRNA vectors (HuSH, Origene, Rockville, MD) were propagated using High Purity Plasmid Midiprep columns (Origene, Rockville, MD), and resuspended in DNAse, RNAse- free water (Sigma, St. Louis, MO). On the day of the surgery, plasmids were complexed with In Vivo Jet-PEI (Polyplus, France) and injected bilaterally into the prelimbic area ( $0.6 \mu \mathrm{L} / \mathrm{side})$ for cohorts of rats subjected to electrophysiological and behavioral studies.

Assessment of PV downregulation

A separate cohort of rats injected unilaterally with shRNA at P34-38 were perfused after P65 with cold saline followed by $4 \%$ PFA in 0.1M PB. Brains were post-fixed for $24 \mathrm{~h}$ and kept in $30 \%$ sucrose/PB. At least six coronal sections $(50 \mu \mathrm{m})$ anterior and six posterior to the injection site were utilized for PV immunohistochemistry as described before [33]. Images were acquired with a Nikon Eclipse Ni-E microscope (Nikon Instruments Inc., Melville, NY) using a 10x objective and analyzed using Image J.

In vivo recordings of local field potential (LFP) in the PFC Changes in the pattern of LFP at 10,20 , and $40 \mathrm{~Hz}$ were determined as previously described [36, 37]. In another cohort of animals, a third bipolar concentric electrode was placed in the basolateral amygdala (BLA) to determine the effect of highfrequency stimulation (HFS)-induced facilitation of LFP in the PFC. After a period of stable baseline recording, a protocol of HFS consisting of four trains of 50 pulses/each at $100 \mathrm{~Hz}$ was delivered into the BLA as previously described [38]. Changes in the slope of the evoked LFP were measured after HFS. At minute 45, a second set of HFS was delivered into the ventral hippocampus and changes in amygdalar-evoked LFP were recorded for an additional $45 \mathrm{~min}$. Each data point was computed by averaging the slope value of eight evoked LFPs from a 2 min window.

Ex vivo recordings of inhibitory synaptic currents in the PFC All procedures were conducted as previously described [39, 40]. For each neuron, the mean inhibitory postsynaptic currents (IPSC) frequency was compared. In another set of neurons, locallyevoked IPSC was elicited by a teflon-coated bipolar electrode placed $\sim 200 \mu \mathrm{m}$ from the cell body along the apical dendrite. The stimulation intensity was titrated to elicit a monosynaptic IPSC response with a failure rate of $\sim 50 \%$ using a paired-pulse protocol to reveal changes in the probability of neurotransmitter release.

Concurrent recordings of excitatory and inhibitory synaptic currents in the PFC

All recordings were conducted using an aCSF free of glutamate and GABA blockers, and a low-chloride-based internal solution to enable concurrent acquisition of excitatory and inhibitory synaptic currents at a single-cell level as previously described [40].
Ex vivo recordings of fast-spiking interneurons in the PFC All recordings were obtained from layer $\mathrm{V}$ using a potassiumbased internal solution, and changes spontaneous excitatory postsynaptic current events were compared as previously described [33]. Only cells that remained stable for at least 20 min after obtaining the whole-cell configuration were included.

\section{Trace fear conditioning and extinction}

We adapted a fear conditioning protocol modified from Zhang \& Rosenkranz [41]. The trace fear conditioning phase consisted of a $120 \mathrm{~s}$ habituation period followed by five pairings of a neutral tone paired with a foot shock $(0.4 \mathrm{~mA})$ a delay of $20 \mathrm{~s}$ from the end of the tone. Conditioning trials were presented using a pseudorandom inter-trial interval of 240-280 s. After conditioning, rats were returned to their home cage for $24 \mathrm{~h}$. Extinction trials began the next day in a visually and tactilely distinct chamber. Following a $120 \mathrm{~s}$ habituation period, the conditioned tone $(20 \mathrm{~s})$ was presented 14 times (60 s per trial) without foot shock to enable extinction of the fear memory. The acquisition of fear extinction is typically revealed by the level of conditioned freezing to the tone that diminishes over repeated trials. An infrared camera connected to the ANY-maze behavioral analysis software (Stoelting Co., IL) was used to record and quantify the time spent freezing per trial.

\section{RESULTS}

PV downregulation diminishes GABAergic transmission

Unlike previous genetic models where PV is completely ablated in the brain [42], we sought to retain peri-adolescent levels of the protein to assess whether the upregulation of PV occurring in the PFC during adolescence was necessary for normal adult functions. In order to prevent the upregulation of PV expression observed in adolescence, we injected an shRNA against PV into the medial PFC during postnatal days (P) 34-38 (Fig. 1a). All analyses of PV expression were compared with the scrambled (Scr)-shRNA control at $\sim$ P65 (Fig. 1a). Using this approach, we were able to achieve $\sim 25 \%$ reduction in PV expression in the injected site compared with the contralateral side, as measured by fluorescence immunohistochemistry (Fig. 1b). The effect was noticeable in the neuropil, due to a reduction of PV-positive innervation, and in individual cells (Fig. 1c). PV knock-down also changed the appearance of "baskets", the PV-positive structures circumscribing the cell body of pyramidal neurons. While still present, these profiles displayed a marked reduction in the intensity of surrounding terminals exemplified by the heat plots (Fig. 1c).

Concomitant with the upregulation of PV expression observed from juveniles to adults [33], there is an increase in GABAergic transmission in the PFC as revealed by the number of spontaneous inhibitory postsynaptic current (IPSC) onto layer $\mathrm{V}$ pyramidal neurons [39]. Thus, we determined if the loss of PV achieved with the shRNA strategy had an effect on GABAergic transmission impinging upon pyramidal neurons. Whole-cell patch-clamp recordings obtained from PFC brain slices revealed that a reduction of PV expression below adult levels is sufficient to decrease the frequency of IPSC onto layer $V$ pyramidal neurons (Fig. 1d) without altering the mean IPSC amplitude (Scr-shRNA: $15.9 \pm 1.2 \mathrm{pA}$; PV-shRNA: $15.4 \pm 0.8 \mathrm{pA}$ ). Moreover, the mean IPSC frequency obtained in the adult PFC of PV-shRNA-treated rats closely resembled that of peri-adolescent animals [39]. Data obtained from locally-evoked IPSC using a protocol of pairedpulse stimulation to elicit monosynaptic responses further revealed a presynaptic mechanism of GABAergic disruption (Fig. 1e). While pyramidal neurons recorded from $\mathrm{Scr}$ controls exhibited a typical paired-pulse suppression (IPSC ${ }_{2} / \mathrm{IPSC}_{1}<1.0$ ), a paired-pulse facilitation (IPSC $2 / \mathrm{IPSC}_{1}>1.0$ ) emerged following PV downregulation (Fig. 1e). This increase in IPSC paired-pulse ratio indicates that the probability of GABA release is decreased. 
a

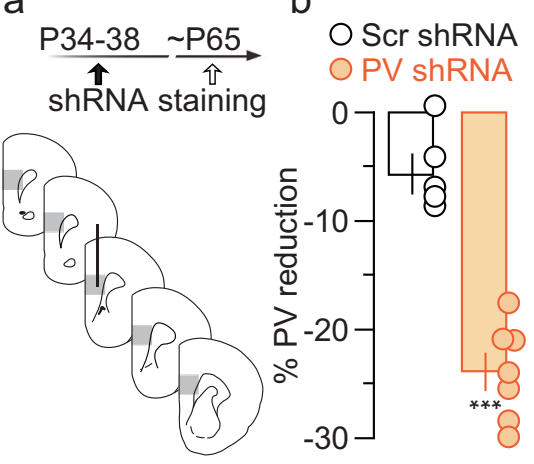

C control side Scr shRNA

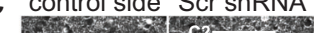

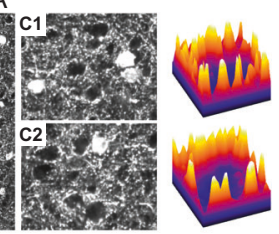

control side PV shRNA
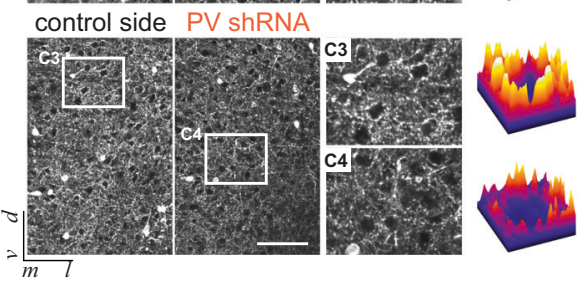
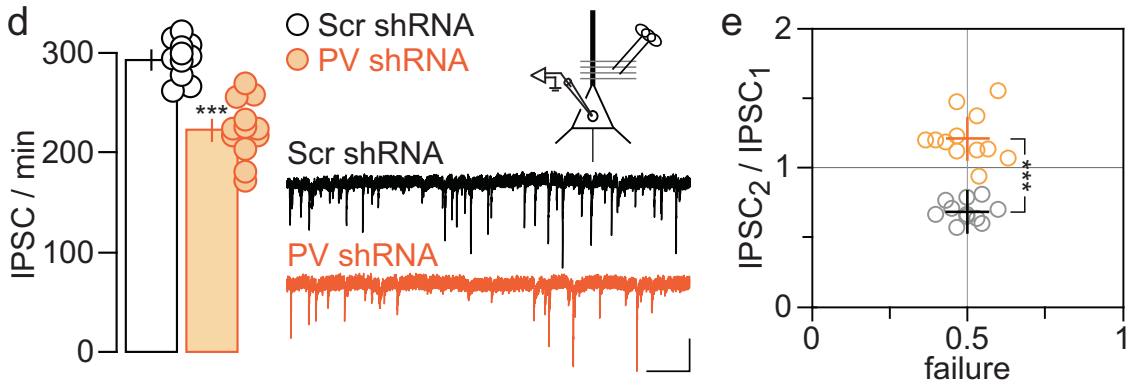

Fig. 1 PV downregulation modifies baskets and reduces GABAergic transmission in the PFC. a Diagram of experimental design for quantification of PV downregulation after unilateral, intra-PFC delivery of scrambled $(\mathrm{Scr} ; n=5)$ or PV shRNA $(n=7)$ during postnatal days (P) 34-38. Anti-PV staining was performed when rats reached $\sim$ P65 in $50 \mu \mathrm{m}$-thick sections $300 \mu \mathrm{m}$ anterior and posterior to the injection site. b Relative to the uninjected side, a $\sim 25 \%$ reduction of PV immunoreactivity can be measured in the PFC 30 days after PV shRNA injection $\left({ }^{* * *} p<0.0005\right.$, unpaired $t$-test). c Representative images of Scr and PV shRNA-treated PFC displaying a pronounced reduction of PV expression in the neuropil and deep layer "baskets" (scale bar: $100 \mu \mathrm{m}$ ). Magnified insets (C1-C4) showing the degree of PV downregulation in deep layer baskets. Right panels: intensity heat plots of individually magnified baskets. d Bar-graph summarizing the effect of PV shRNA treatment on spontaneous inhibitory postsynaptic current (IPSC) recorded in the PFC at P65-85. Whole-cell patch-clamp recordings from layer V pyramidal neurons revealed a marked reduction of IPSC frequency (events/min) in the PV shRNA group (12 cells, 6 rats) compared with Scr controls (11 cells, 6 rats; ${ }^{* * *} p<0.0001$, unpaired $t$-test). Inset are examples traces of spontaneous IPSC recorded from layer $V$ pyramidal neurons illustrating the effect of PV shRNA in the PFC (calibration: 15pA, 1s). e Summary of the data obtained from layer V pyramidal neurons using a paired-pulse protocol of minimal stimulation at $50 \mathrm{~ms}$ interval. Note that the intensity of stimulation was titrated to elicit monosynaptic IPSC responses at $\sim 50 \%$ failure rate in both groups to enable the detection of any changes in the probability of GABA release. While pyramidal neurons recorded from Scr controls (10 cells, 5 rats) exhibited IPSC $2 / I P S C_{1}$ ratios $<1.0$, all neurons recorded from the PV-shRNA group (13 cells, 7 rats) showed IPSC $2 / I_{P S C}$ ratios $>1.0$ (*** $p<0.001 \mathrm{vs.} \mathrm{Scr} \mathrm{shRNA,} \mathrm{unpaired} t$-test), indicating that the probability of GABA release is decreased following PV downregulation.

Altogether, these data indicate a presynaptic mechanism underlies the observed GABAergic deficit and demonstrate that the developmental upregulation of PV during adolescence is necessary to sustain normal levels of GABAergic transmission in the adult PFC.

PV downregulation disrupts the excitatory-inhibitory balance in the PFC

The negative impact of PV downregulation on GABAergic transmission is likely to alter the balance of excitatory-inhibitory (E-I) activity in the PFC only if local glutamatergic transmission remains unaltered. To test this hypothesis, we conducted wholecell patch-clamp recordings from layer $\mathrm{V}$ pyramidal neurons using a protocol that enables the acquisition of GABAergic and glutamatergic synaptic activity within a single cell (Fig. 2a; [40]). The results showed both Scr- and PV-shRNA-treated PFC display nearly identical levels of postsynaptic current events recorded at $-60 \mathrm{mV}$ (PSC $-60 \mathrm{mv}$; Fig. 2b), indicating PV-shRNA treatment does not affect the activity of glutamatergic synapses. In contrast, PV downregulation selectively diminished the GABAergic component of synaptic activity, as revealed by a marked reduction in PSC $+15 \mathrm{mV}$ frequency (Fig. 2b). Consequently, a higher E/I ratio emerged in the PFC of PV-shRNA-treated animals (Fig. 2c). Notably, such a deficit was already detectable by P45, a time when adult levels of GABAergic activity are already attained (Fig. 2b). Further analyses revealed the increase in $\mathrm{E} / \mathrm{I}$ ratio is correlated with the frequency of IPSC (Fig. 2d), indicating that the E-I imbalance induced results from a preferential disruption of PFC GABAergic transmission. Collectively, these results demonstrate that a modest downregulation of $\mathrm{PV}$ expression can impact the E/I ratio of PFC output neurons, which in turn could have a detrimental effect in signal processing and integration.

PV downregulation prevents the normal facilitation of glutamatergic transmission onto $\mathrm{FSI}$

The E-I imbalance observed in the PFC following adolescent PV downregulation could arise from a developmental deficit in the recruitment of fast-spiking interneurons (FSI) by excitatory inputs during adolescence [33]. To test this hypothesis, we conducted electrophysiological recordings to determine whether the characteristic facilitation of glutamatergic transmission onto FSI observed during adolescence [33] is disrupted following PV downregulation. FSI were identified by a non-adapting firing response to somatic depolarization and a prominent afterhyperpolarization potential as previously described [33]. Relative to Scr-shRNA controls, FSI recorded from the PV-shRNA-treated group showed a lower frequency of excitatory postsynaptic currents (EPSC) (Fig. 2f-h) without any detectable changes in the mean EPSC amplitude (Scr-shRNA: $15.8 \pm 1.2 \mathrm{pA}$; PV-shRNA: $15.4 \pm 0.8 \mathrm{pA}$ ). Such a deficit in EPSC transmission was detectable 
a

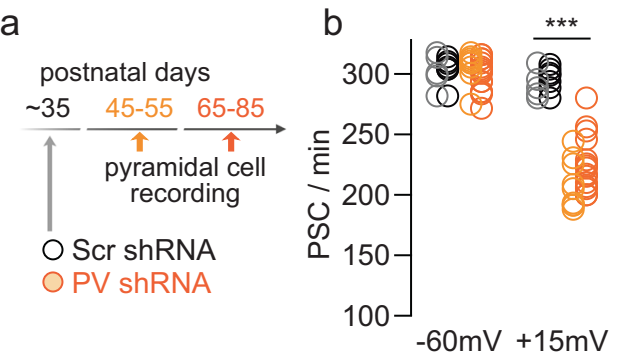

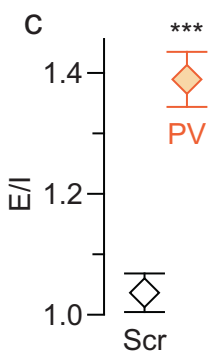

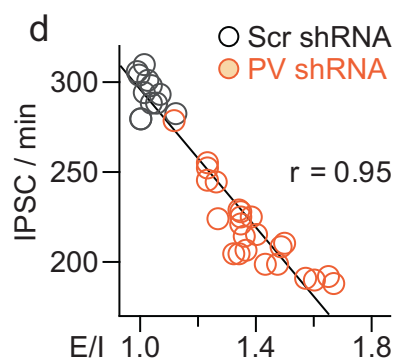

e

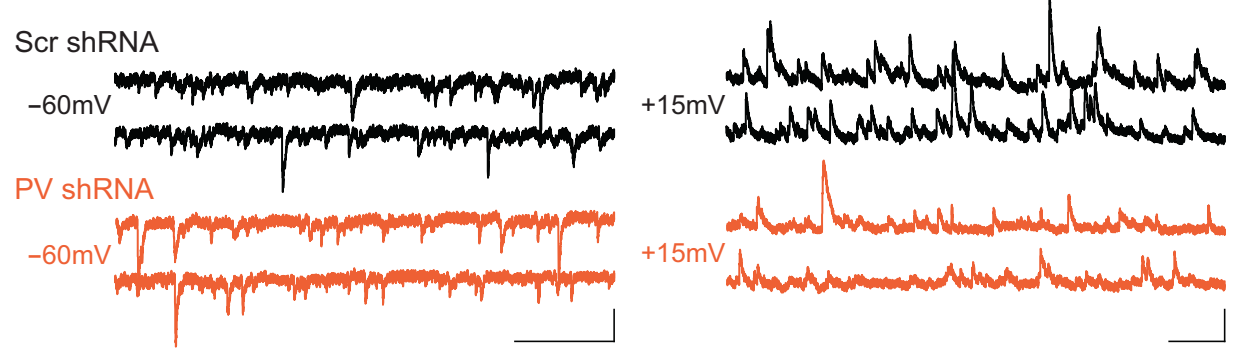

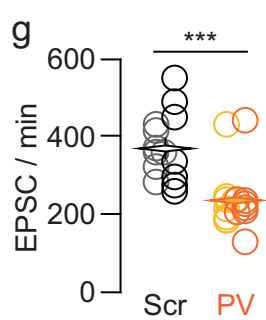

PV shRNA

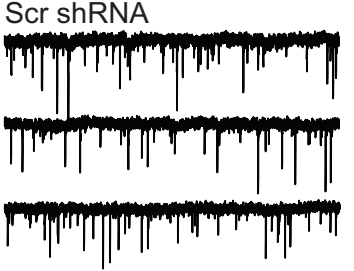

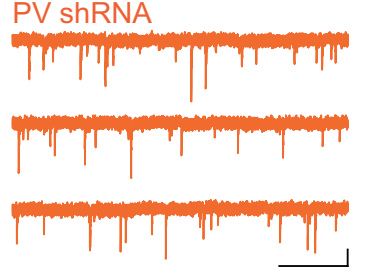

Fig. 2 Downregulation of PV increases the ratio of excitatory-inhibitory synaptic activity onto layer $\mathbf{V}$ pyramidal neurons and reduces the frequency of excitatory transmission onto fast-spiking interneurons. a Changes in excitatory (E)-inhibitory (I) balance of synaptic activity following intra-PFC delivery of Scr or PV shRNA at P35 were determined at P45-55 and P65-85. Data from the P45-55 group were collected to assess whether the functional impact of PV shRNA is already detectable at 10-15 days post-delivery. b All recordings were obtained from layer $\mathrm{V}$ pyramidal neurons in the PFC using a low-chloride-based internal solution that enables concurrent acquisition of inhibitory (PSC $+15 \mathrm{mv}$ ) and excitatory (PSC $-60 \mathrm{mv}$ ) postsynaptic currents (see Materials \& Methods). Relative to Scr controls (P45-55: gray circles, 4 rats; P65-85: black circles, 5 rats), PV shRNA delivery did not alter the frequency of PSC 60 mv events (PSC/min) at P45-55 (yellow circles, 4 rats) or P65-85 (orange circles, 5 rats). In contrast, intra-PFC delivery of PV shRNA diminished the frequency of PSC $+15 \mathrm{mv}$ in layer V pyramidal neurons, an effect that can be detected at P45 (*** $p<0.0005$, unpaired $t$-test). c Calculation of the E/I ratio for each individual neuron recorded from P45-55 and P65-85. Data from both age groups were pooled. Relative to Scr controls, treatment with PV shRNA markedly increased the E/I ratio in layer $V$ pyramidal neurons by more than $30 \%\left({ }^{* *} p<0.0005\right.$, unpaired $t$-test). d Further analysis revealed a significant correlation ( $p$ $<0.001$ ) between the E/I ratio and the frequency of PSC $+15 \mathrm{mv}$ (i.e., IPSC) events. e Example traces of PSC $+15 \mathrm{mv}$ and PSC $-60 \mathrm{mv}$ recorded from layer V pyramidal neurons illustrating the effect of intra-PFC delivery of PV shRNA shown in b (calibration: $25 \mathrm{pA} / 0.5 \mathrm{~s}$ for PSC $-60 \mathrm{mv}$ and 40 pA/0.5 s for PSC $+15 \mathrm{mv}$ ). f Impact of intra-PFC delivery of PV shRNA ( P35) on fast-spiking interneurons' (FSI) excitatory postsynaptic (EPSC) events recorded at P45-55 and P65-85. g Relative to the Scr shRNA control group (P45-55: 4 rats; P65-85: 5 rats), a marked reduction in EPSC frequency was observed in FSI following adolescent PV downregulation (P45-55: 4 rats; P65-85: 5 rats; ${ }^{* * *} p<0.0001$, unpaired $t$-test). h Example traces of spontaneous EPSC recorded from FSI illustrating the impact of adolescent delivery of PV shRNA into the PFC shown in (f) (calibration: $10 \mathrm{pA}, 500 \mathrm{~ms}$ ).

by P45 (Fig. 2g, h), a time when adult levels of glutamatergic activity onto FSI are already attained [33]. Notably, the level of EPSC frequency observed following adolescent PV downregulation resembles that of naïve juvenile animals [33]. Of note, PV downregulation did not alter the excitability of FSI (resting membrane potential, $\mathrm{mV}$ : $65.6 \pm 0.7$ vs. $66.7 \pm 1.0$; input resistance, mOhm: $323.9 \pm 26.2$ vs. $323.2 \pm 25.5$; after-hyperpolarization, $\mathrm{mV}$ : $19.9 \pm 1.2$ vs. $19.7 \pm 1.5$; Scr-shRNA vs. PV-shRNA, respectively). Together, these results indicate that adult levels of PV expression are necessary for sustaining normal levels excitatory transmission onto FSI.

Disruption of hippocampal-PFC transmission following PV downregulation

To assess whether prefrontal processing of afferent signal is disrupted by local downregulation of PV expression, we conducted local field potential (LFP) recordings and examined the pattern of PFC responses to ventral hippocampal train stimulation at 10,20 , and $40 \mathrm{~Hz}$. The ventral hippocampus is one of the main regions projecting to the PFC and its activation can reveal distinct developmental stages of prefrontal GABAergic function in vivo $[37,39,43]$. Using this approach, we found the normal pattern of LFP response at $10 \mathrm{~Hz}$ remained unaltered in the PFC of PV-shRNA-treated rats (Fig. 3a, e, f). At $20 \mathrm{~Hz}, \mathrm{Scr}-$ shRNA animals showed the typical transient LFP suppression observed in the PFC of normal adults $[36,37]$, whereas the PVshRNA-treated group displayed a pattern of LFP facilitation (Fig. 3b, e, f). Similarly, the level of LFP suppression upon hippocampal stimulation at $40 \mathrm{~Hz}$ was smaller in the PFC of PVshRNA-treated rats relative to Scr-shRNA controls (Fig. 3c, e, f). Of note, the patterns of LFP facilitation (at $20 \mathrm{~Hz}$ ) and attenuated suppression (at $40 \mathrm{~Hz}$ ) observed following PV downregulation were indistinguishable from the responses obtained in juveniles (Fig. 3e; see also [37]) and those recorded in adults following PFC blockade of GABA-AR transmission [36, 37]. These results indicate that an optimal level of PV expression is needed to enable PFC GABAergic control of high-frequency afferent signal from the ventral hippocampus. Thus, the ability of PV-positive 

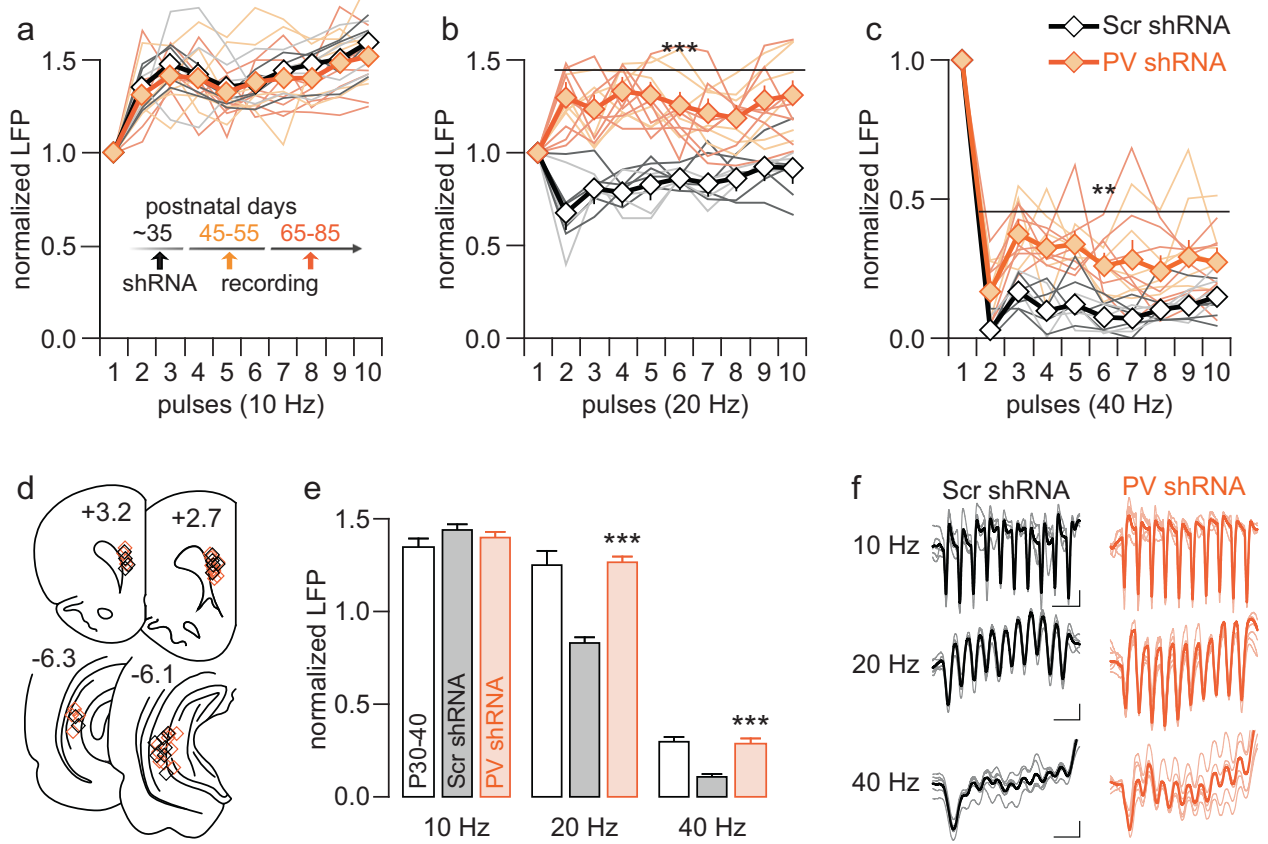

Fig. 3 Decreased levels of PV in the prefrontal cortex confer juvenile-like pattern of hippocampal-evoked response. a The pattern of ventral hippocampal-evoked $(10 \mathrm{~Hz})$ facilitation of local field potential $(\mathrm{LFP})$ responses in the PFC was indistinguishable between Scr $(n=9)$ and PV shRNA $(n=13)$ groups. b Following hippocampal stimulation at $20 \mathrm{~Hz}$, a normal transient suppression of LFP response was recorded in the PFC of Scr controls (P45-55: gray lines, 4 rats; P65-85: black lines, 5 rats) whereas a pattern of prefrontal LFP facilitation emerged in PV shRNA-treated animals (P45-55: yellow lines, 6 rats; P65-85: orange lines, 7 rats; treatment $x$ pulse interaction, $F_{9200}=6.1$, **** $p<0.0001 ;$ main treatment effect, $F_{1200}=349.9, p<0.0001$, two-way ANOVA). Note the abnormal facilitation of LFP response in the PFC of PV shRNA-treated animals is already apparent at P45 (yellow lines). c At $40 \mathrm{~Hz}$, both Scr and PV shRNA groups exhibited similar patterns of LFP suppression in the PFC. However, the magnitude of prefrontal LFP suppression was markedly reduced following PV shRNA delivery (treatment $\times$ pulse interaction, $F_{9200}=2.6,{ }^{* *} p<0.005$; main treatment effect, $F_{1200}=147.1, p<0.0001$, two-way ANOVA). Such a disruption was also apparent at P45 (yellow lines). d Diagram of PFC (top) and hippocampal (bottom) coronal sections showing the placement for all recording and stimulating electrodes, respectively (black: Scr shRNA group; orange: PV shRNA group). e Bar-graph summarizing the impact of PV shRNA on prefrontal LFP responses presented in panels $(\mathbf{a}-\mathbf{c})$. Data from a cohort of naïve P30-40 rats $(n=7)$ were included for comparison. Note that the levels of LFP facilitation (at $20 \mathrm{~Hz}$ ) and LFP suppression (at $40 \mathrm{~Hz}$ ) recorded in the PFC of PV shRNA-treated group are equivalent to those observed in P30-40 animals ( ${ }^{* * *} p<0.0005$ vs. P30-40 or PV shRNA, Tukey post-hoc test after significant one-way ANOVA; $F_{226}=40.3, p<$ 0.0001 for $20 \mathrm{~Hz}$ and $F_{226}=37.1, p<0.0001 \mathrm{for} 40 \mathrm{~Hz}$ ). f Example traces of prefrontal LFP response to hippocampal train stimulation at 10 , 20, and $40 \mathrm{~Hz}$ utilized for the analysis in panels (a-c) (calibration: $3 \mathrm{mV} / 200 \mathrm{~ms}$ at $10 \mathrm{~Hz} ; 3 \mathrm{mV} / 100 \mathrm{~ms}$ at $20 \mathrm{~Hz} ; 6 \mathrm{mV} / 50 \mathrm{~ms}$ at $40 \mathrm{~Hz}$ ).

interneurons to handle high-frequency afferent drive relies in part on the expression of PV itself.

PV downregulation prevents the normal modulation of basolateral amygdala inputs by the ventral hippocampus in the PFC

The enduring $\mathrm{E}-\mathrm{I}$ imbalance resulting from PV downregulation in the PFC could also disrupt the developmentally-regulated modulation of basolateral amygdala (BLA) inputs by the ventral hippocampus [38]. To test this hypothesis, we employed a protocol of high-frequency stimulation (HFS; Fig. 4) to induce potentiation of prefrontal LFP elicited from the BLA. Following potentiation of the BLA-evoked response, a second set of HFS was delivered into the ventral hippocampus to determine whether the degree of prefrontal depotentiation is disrupted by the PV shRNA treatment. We found the pattern of LFP potentiation resulting from HFS of the BLA remained unaltered following PV downregulation in the PFC (Fig. 4a, b). This is consistent with previous data showing that plasticity of the BLA-to-PFC pathway does not rely on prefrontal GABAergic transmission [43]. However, the characteristic depotentiation of BLA-evoked LFP upon sequential HFS of the ventral hippocampus was only observed in the PFC of Scr-shRNA controls (Fig. 4b). In PV-shRNA animals, hippocampal HFS failed to disrupt the potentiated BLA-evoked LFP in the PFC (Fig. 4b, c), a pattern of response identical to that observed in naïve juveniles [38]. Together, these results support the premise that an optimal level of PV expression in the PFC is needed to enable the inhibitory control of BLA inputs by the ventral hippocampus.

PV downregulation impairs the level of extinction of a trace-fear memory

PFC processing of ventral hippocampal and BLA inputs is critical for the expression of cognitive functions [44, 45] including the acquisition and extinction of conditioned fear memories [46, 47]. In order to test whether PV downregulation in the PFC during adolescence impacts behavioral outcomes in adulthood, we utilized a trace-fear conditioning paradigm in which the cue (tone) and shock are separated by a $20 \mathrm{~s}$-delay. This delay engages the hippocampalPFC pathway and provides a behavioral readout of functional connectivity between the PFC, ventral hippocampus, and BLA [46].

Compared with Scr controls, bilateral downregulation of PV in the PFC does not disrupt the acquisition of cue-mediated fear response tested in adulthood (Fig. 5a). In fact, the behavioral response obtained from Scr- and PV-shRNA-treated groups were indistinguishable from each other, achieving more than $80 \%$ of freezing after 5 tone-shock presentations (Fig. 5a). Similarly, both groups exhibited similar levels of baseline freezing during habituation prior to extinction on day 2 (Scr-shRNA: $11.5 \pm 1.8 \%$ vs. PV-shRNA: $11.4 \pm 1.4 \%$ ). During testing for the extinction of the fear memory, the Scr-shRNA group showed the typical gradual reduction of the freezing response to the tone over repeated trials (Fig. 5b). In contrast, the PV-shRNA group displayed increased 
a

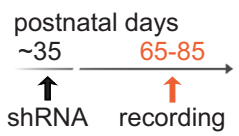

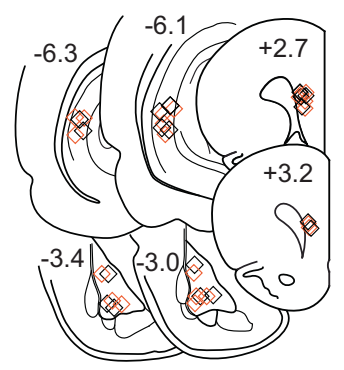

b

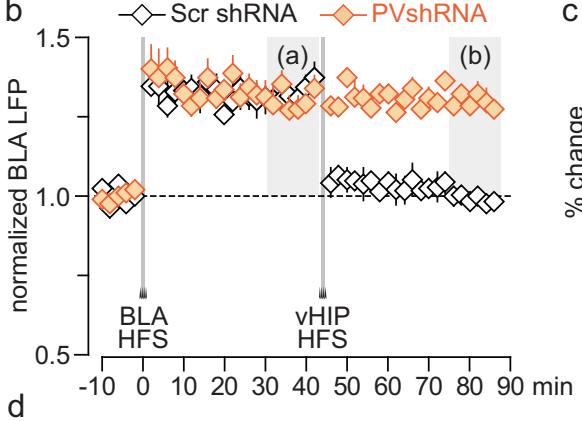

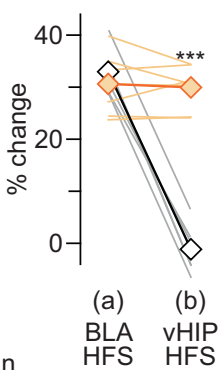

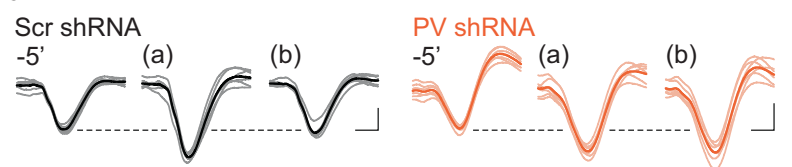

Fig. 4 PV downregulation impairs the inhibitory control of amygdalar inputs to the prefrontal cortex by the ventral hippocampus. a Timeline of the experimental design and diagrams of brain coronal sections showing the location for all PFC recording sites and placement of ventral hippocampal (vHIP) and basolateral amygdalar (BLA) stimulating electrodes used to collect the data shown in (b) (black: Scr shRNA group; orange: PV shRNA group). b PFC downregulation of PV did not alter the typical potentiation of BLA-evoked LFP induced by a protocol of high-frequency stimulation in the BLA (HFS; see Materials \& Methods). However, only the Scr shRNA group showed the normal response to vHIP HFS applied $40 \mathrm{~min}$ post-BLA potentiation. While HFS of the vHIP resets the LFP facilitation driven by the BLA in Scr controls ( $n=6$ rats), the suppression of BLA-evoked LFP response is no longer observed in PV shRNA-treated animals $(n=7$ rats). c Summary of the mean LFP response obtained from the last $10 \mathrm{~min}$ post-HFS of the BLA (a) and vHIP (b) shown in (b) (area marked in gray). Two-way ANOVA revealed main effects of input stimulation $\left(F_{122}=43.6, p<0.0001\right)$, PFC shRNA treatment $\left(F_{122}=29.7, p<0.0005\right)$, and input $\times$ treatment interaction $\left(F_{122}=39.9, p<0.0001 ;{ }^{* * *} p<0.0005\right.$ vs. Scr shRNA, Tukey post-hoc test). d Traces of BLA-evoked LFP responses recorded from the PFC illustrating the impact of PV shRNA shown in (b) (-5': baseline; (a): post-BLA HFS; (b) post-vHIP HFS; calibration: $5 \mathrm{mV}, 20 \mathrm{~ms})$.
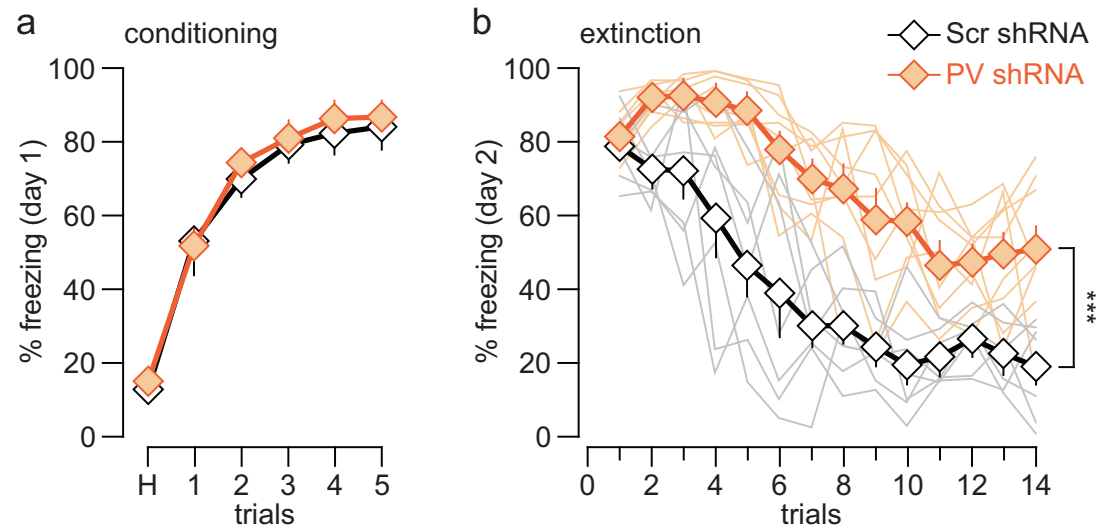

Fig. 5 Downregulation of PV in the prefrontal cortex increases the level of freezing response during extinction testing. a $A$ "trace" fear conditioning paradigm in which the cue (tone) and the shock $(1 \mathrm{~s}, 0.4 \mathrm{~mA})$ are separated by $20 \mathrm{~s}$ was utilized to measure intact hippocampalPFC connectivity. Following habituation $(\mathrm{H})$ on day 1 , both Scr-shRNA ( $n=7$ rats) and PV-shRNA ( $n=9$ rats) groups were conditioned to the tone as evidenced by increasing levels of freezing over five trials, with no differences in the rate of acquisition of the fear response. $\mathbf{b}$ Twentyfour hours later (day 2), extinction of the cue-induced freezing response was measured in both experimental groups. Relative to Scr controls, PV shRNA-treated rats exhibited a slower extinction rate (main effect of treatment, $F_{1196}=192.2,{ }^{* * *} p<0.0005$; treatment $\times$ trial interaction, $F_{13196}=2.0, p=0.024$, two-way ANOVA).

freezing times relative to Scr controls, a difference which persisted until the last trial of cue presentation (Fig. 5b). Post-hoc analyses also revealed a difference in the level of freezing between the first and last trials of extinction for both Scr- and PV-shRNA-treated rats (Scr-shRNA: $p<0.00005$, PV-shRNA: $p<0.01$, Tukey post-hoc test). Altogether, these results indicate that an optimal level of PV expression in the PFC is critical for regulating the level of extinction learning.

\section{DISCUSSION}

While genetic ablation of PV-positive interneuron activity has provided proof of concept data on the importance of prefrontal PV interneurons in cognitive functions [48-53], it has yet to provide plausible biological mechanisms to explain the reduction of PV-positive interneuron activity commonly described in psychiatric disorders. Using an shRNA approach at the time PV expression is about to rise, we were able to detect the functional impact of PV-shRNA as early as 10 days post-delivery. The results presented herein show that a mere downregulation of PV to adolescent levels is sufficient to decrease GABAergic transmission and disrupt the $\mathrm{E}-\mathrm{I}$ balance in the PFC, ultimately reducing the inhibitory control of afferent integration to levels indistinguishable from those in juvenile animals. The defect in afferent information processing is further manifested as an impairment in the level of extinction learning. Altogether, these findings provide a novel, biologically-relevant mechanism by which GABAergic transmission in the PFC can be regulated during adolescent development.

The upregulation of $\mathrm{PV}$ in multiple brain regions at specific time points $[14,17,54,55]$ is seemingly part of a developmental 
program triggered by increased synaptic activity from specific inputs. Accordingly, PV expression in sensory cortices is dramatically reduced after blocking afferent drive [23-25, 30-32]. It remains to be defined whether a single or concerted glutamatergic activity from PFC afferent structures (e.g., ventral hippocampus, BLA, or mediodorsal thalamus) is responsible for eliciting the genetic/epigenetic program that results in PV upregulation within the developmental window studied here. Previous work in organotypic cultures suggests such a program may be established early on and may vary for each cortical layer [26,56]. Interestingly, genetic ablation of the global transcriptional activator PGC-1a results in a striking loss of cortical PV among other proteins specifically associated with GABAergic interneurons [57, 58]. Nonetheless, our study indicates that preventing the developmental increase of PV alone is sufficient to halt the gain of prefrontal GABAergic transmission attained during adolescence, suggesting that even small changes in PV concentration in the PFC can affect local inhibitory control and disrupt its optimal computational capacity [59].

The continued increase in PV expression observed in the PFC during adolescence serves an integral role in GABA neurotransmission in adulthood possibly by allowing PV-positive interneurons tighter temporal control over inputs and/or by conferring increased calcium buffering capacity to sustain GABA release [11]. Irrespective of the synaptic mechanisms by which PV regulates GABAergic transmission, loss of PV results in a net increase in the $E / I$ ratio, likely through the inability of GABAergic interneurons to sustain a high firing rate during periods of high cognitive demand. Such deficiencies would not be apparent until the protracted consolidation of long and short-term inputs occurring in the PFC during adolescence $[36,37,59]$. In support of this idea, previous work has demonstrated that a complete absence of PV preferentially alters synaptic activity of narrowly timed events $[42,60,61]$, indicating PV function only becomes manifest upon certain type of inputs defined by their proximity and/or frequency. Our results expand this concept to demonstrate that only mature levels of cortical PV can exert proper inhibitory control of afferent drive received at high frequencies (i.e., $>20 \mathrm{~Hz}$ ). It remains to be understood why PV downregulation selectively affects PFC integration of hippocampal inputs, despite the amygdala also targets PV interneurons in the PFC $[62,63]$. The input-specific impact of PV downregulation suggests that there are concurrent, perhaps conditional mechanisms in the PFC and hippocampus that are required for normal development and function of both structures. Supporting this idea, the ventral hippocampus also undergoes a protracted maturation in its PFC-projection domain during adolescence $[64,65]$. Thus, the amount of PV within an interneuron needs to match the strength of input-specific glutamatergic transmission in order to enable an optimal synaptic response with sufficient temporal control of afferent drive.

Not surprisingly, the results presented herein diverge from the ones obtained in full PV knock-out mice $[60,61,66]$. Complete absence of PV has been reported to enhance the excitability of FSI [66] and facilitate GABAergic transmission as revealed by an increased in paired-pulse ratio [60]. Of note, these studies did not employ protocols of minimal stimulation to reveal presynaptic changes in transmitter release [67], leaving open the possibility that the increased paired-pulse ratio observed in full PV knock-out mice could arise from compensatory processes from the absence of PV since embryonic stages, thus rendering FSI hyperexcitable [66]. In contrast, partial PV downregulation during adolescence in the PFC did not change FSI excitability, but it markedly reduced the frequency of IPSC in conjunction with an increased pairedpulse ratio of evoked IPSC at minimal stimulation, all indicative of a presynaptic deficit in GABA release onto pyramidal neurons.

We propose the full range of PV function is the sum of a concentration and time-dependent increase of PV at different cellular compartments: 1) in dendrites where PV-positive interneurons receive most of their synaptic inputs, and 2) in synaptic terminals, where PV is positioned to regulate neurotransmitter release. The observation that the increase in PV occurs primarily at terminals at critical developmental points (documented as a prevalence of "basket" structures $[18,33])$, suggests that the role of PV in regulating GABA release becomes pronounced only after PV concentration reaches a "critical mass" during development.

Although the retrieval of extinction memory was not tested here, our data indicate that small changes in PV expression in the PFC are sufficient to cause an impairment in the level of extinction learning. Similar alterations in neural circuits regulating extinction behavior have been documented in schizophrenia [68], and recapitulated in animal models of the disease [69]. Interestingly, a small but consistent reduction in prefrontal PV expression ( 20\%) has been observed in schizophrenia [35, 70], suggesting that reduced PV levels may explain some, but certainly not all the pathophysiology of the disease in which more than one brain structure is compromised. Whether the reduction in PV observed in schizophrenia is the result of increased oxidative stress [71, 72], a lack of developmental upregulation [1], or a combination of both, a mismatch between prefrontal PV expression and afferent drive is enough to render the PFC hypofunctional. Future studies are warranted to determine the extent of behavioral changes resulting from such a discrete disruption of PV expression in the PFC during adolescence.

\section{CONCLUSIONS}

The experimental observations presented above together with the computational model proposed by Eggermann and Jonas [73], indicate that the ultimate role of PV can only be examined and understood under a developmental "lens" as its function is highly dependent on the absolute amount present at any given developmental window, which itself is a function of afferent activity. In light of the results presented here, a re-evaluation of the role of PV based on the developmental status of each region studied is warranted. Our results provide unequivocal demonstration that the protracted expression of PV in the PFC during adolescence is a biologically relevant process that must take place to attain mature levels of inhibitory transmission and support adult PFC-dependent behaviors.

\section{FUNDING AND DISCLOSURE}

This work was supported by grants R01-MH086507 and R01$\mathrm{MH} 105488$ to KYT. The authors have no financial disclosures or conflict of interest to disclose.

\section{ACKNOWLEDGEMENTS}

We thank Ms. Yuliya Zayats for assistance with tissue processing. This project was initiated at Rosalind Franklin University-Chicago Medical School.

\section{AUTHOR CONTRIBUTIONS}

$A C$ and KYT designed the study, wrote the paper, and prepared the figures. AC, EFB, and DRT performed the experiments and analyzed the data under supervision of KYT.

\section{ADDITIONAL INFORMATION}

Supplementary Information accompanies this paper at (https://doi.org/10.1038/ s41386-020-0709-9).

Publisher's note Springer Nature remains neutral with regard to jurisdictional claims in published maps and institutional affiliations. 


\section{REFERENCES}

1. Caballero A, Tseng KY. GABAergic function as a limiting factor for prefrontal maturation during adolescence. Trends Neurosci. 2016;39:441-48.

2. Giedd JN, Blumenthal J, Jeffries NO, Castellanos FX, Liu H, Zijdenbos A, et al. Brain development during childhood and adolescence: a longitudinal MRI study. Nat Neurosci. 1999;2:861-3.

3. Gogtay N, Giedd JN, Lusk L, Hayashi KM, Greenstein D, Vaituzis AC, et al. Dynamic mapping of human cortical development during childhood through early adulthood. Proc Natl Acad Sci USA. 2004;101:8174-9.

4. Caballero A, Granberg R, Tseng KY. Mechanisms contributing to prefrontal cortex maturation during adolescence. Neurosci Biobehav Rev. 2016;70:4-12.

5. Paus T, Keshavan M, Giedd JN. Why do many psychiatric disorders emerge during adolescence? Nat Rev Neurosci. 2008;9:947-57.

6. Volk DW, Lewis DA. Early developmental disturbances of cortical inhibitory neurons: contribution to cognitive deficits in schizophrenia. Schizophr Bull 2014;40:952-7.

7. Celio MR. Calbindin D-28k and parvalbumin in the rat nervous system. Neuroscience 1990;35:375-475.

8. Schwaller B. Cytosolic Ca2+ buffers. Cold Spring Harb Perspect Biol. 2010;2: a004051.

9. Chard PS, Bleakman D, Christakos S, Fullmer CS, Miller RJ. Calcium buffering properties of calbindin D28k and parvalbumin in rat sensory neurones. J Physiol. 1993;472:341-57.

10. Lee SH, Schwaller B, Neher E. Kinetics of $\mathrm{Ca} 2+$ binding to parvalbumin in bovine chromaffin cells: implications for $[\mathrm{Ca} 2+]$ transients of neuronal dendrites. J Physiol. 2000;525(Pt 2):419-32.

11. Collin T, Chat M, Lucas MG, Moreno H, Racay P, Schwaller B, et al. Developmental changes in parvalbumin regulate presynaptic $\mathrm{Ca} 2+$ signaling. J Neurosci. 2005;25:96-107.

12. Stuhlfauth I, Reininghaus J, Jockusch $\mathrm{H}$, Heizmann CW. Calcium-binding protein, parvalbumin, is reduced in mutant mammalian muscle with abnormal contractile properties. Proc Natl Acad Sci USA. 1984;81:4814-8.

13. Berchtold MW, Means AR. The Ca2+-binding protein parvalbumin: molecular cloning and developmental regulation of mRNA abundance. Proc Natl Acad Sci USA. 1985;82:1414-8.

14. Solbach S, Celio MR. Ontogeny of the calcium binding protein parvalbumin in the rat nervous system. Anat Embryol (Berl). 1991;184:103-24.

15. Hendrickson AE, Van Brederode JF, Mulligan KA, Celio MR. Development of the calcium-binding protein parvalbumin and calbindin in monkey striate cortex. J Comp Neurol. 1991;307:626-46.

16. Soriano E, Del Rio JA, Ferrer I, Auladell C, De Lecea L, Alcantara S. Late appearance of parvalbumin-immunoreactive neurons in the rodent cerebral cortex does not follow an 'inside-out' sequence. Neurosci Lett. 1992;142:147-50.

17. Alcantara S, Ferrer I, Soriano E. Postnatal development of parvalbumin and calbindin D28K immunoreactivities in the cerebral cortex of the rat. Anat Embryol (Berl). 1993;188:63-73.

18. del Rio JA, de Lecea L, Ferrer I, Soriano E. The development of parvalbuminimmunoreactivity in the neocortex of the mouse. Brain Res Dev Brain Res. 1994;81:247-59.

19. Conde F, Lund JS, Lewis DA. The hierarchical development of monkey visual cortical regions as revealed by the maturation of parvalbumin-immunoreactive neurons. Brain Res Dev Brain Res. 1996;96:261-76.

20. Reynolds GP, Beasley CL. GABAergic neuronal subtypes in the human frontal cortex-development and deficits in schizophrenia. J Chem Neuroanat. 2001;22:95-100.

21. Erickson SL, Lewis DA. Postnatal development of parvalbumin- and GABA transporter-immunoreactive axon terminals in monkey prefrontal cortex. J Comp Neurol. 2002;448:186-202.

22. Leberer E, Pette D. Neural regulation of parvalbumin expression in mammalian skeletal muscle. Biochem J. 1986;235:67-73.

23. Cellerino A, Siciliano R, Domenici L, Maffei L. Parvalbumin immunoreactivity: a reliable marker for the effects of monocular deprivation in the rat visual cortex. Neuroscience 1992;51:749-53.

24. Blumcke I, Weruaga E, Kasas S, Hendrickson AE, Celio MR. Discrete reduction patterns of parvalbumin and calbindin D-28k immunoreactivity in the dorsal lateral geniculate nucleus and the striate cortex of adult macaque monkeys after monocular enucleation. Vis Neurosci. 1994;11:1-11.

25. Alcantara S, Soriano E, Ferrer I. Thalamic and basal forebrain afferents modulate the development of parvalbumin and calbindin D28k immunoreactivity in the barrel cortex of the rat. Eur J Neurosci. 1996;8:1522-34.

26. Patz S, Grabert J, Gorba T, Wirth MJ, Wahle P. Parvalbumin expression in visual cortical interneurons depends on neuronal activity and TrkB ligands during an Early period of postnatal development. Cereb Cortex 2004;14:342-51.

27. Celio MR, Heizmann CW. Calcium-binding protein parvalbumin as a neuronal marker. Nature 1981;293:300-2.
28. Le Magueresse $C$, Monyer H. GABAergic interneurons shape the functional maturation of the cortex. Neuron 2013;77:388-405.

29. Bartos M, Vida I, Jonas P. Synaptic mechanisms of synchronized gamma oscillations in inhibitory interneuron networks. Nat Rev Neurosci. 2007;8:45-56.

30. Carder RK, Leclerc SS, Hendry SH. Regulation of calcium-binding protein immunoreactivity in GABA neurons of macaque primary visual cortex. Cereb Cortex 1996;6:271-87.

31. Philpot BD, Lim JH, Brunjes PC. Activity-dependent regulation of calcium-binding proteins in the developing rat olfactory bulb. J Comp Neurol. 1997;387:12-26.

32. Jiao Y, Zhang C, Yanagawa Y, Sun QQ. Major effects of sensory experiences on the neocortical inhibitory circuits. J Neurosci. 2006;26:8691-701.

33. Caballero A, Flores-Barrera E, Cass DK, Tseng KY. Differential regulation of parvalbumin and calretinin interneurons in the prefrontal cortex during adolescence. Brain Struct Funct. 2014;219:395-406.

34. Lewis DA. Inhibitory neurons in human cortical circuits: substrate for cognitive dysfunction in schizophrenia. Curr Opin Neurobiol. 2014;26:22-6.

35. Hashimoto T, Volk DW, Eggan SM, Mirnics K, Pierri JN, Sun Z, et al. Gene expression deficits in a subclass of GABA neurons in the prefrontal cortex of subjects with schizophrenia. J Neurosci. 2003;23:6315-26.

36. Cass DK, Thomases DR, Caballero A, Tseng KY. Developmental disruption of gamma-aminobutyric acid function in the medial prefrontal cortex by noncontingent cocaine exposure during early adolescence. Biol Psychiatry 2013;74:490-501.

37. Thomases DR, Cass DK, Tseng KY. Periadolescent exposure to the NMDA receptor antagonist MK-801 impairs the functional maturation of local GABAergic circuits in the adult prefrontal cortex. J Neurosci. 2013;33:26-34.

38. Thomases DR, Cass DK, Meyer JD, Caballero A, Tseng KY. Early adolescent MK-801 exposure impairs the maturation of ventral hippocampal control of basolateral amygdala drive in the adult prefrontal cortex. J Neurosci. 2014;34:9059-66.

39. Cass DK, Flores-Barrera E, Thomases DR, Vital WF, Caballero A, Tseng KY. CB1 cannabinoid receptor stimulation during adolescence impairs the maturation of GABA function in the adult rat prefrontal cortex. Mol Psychiatry 2014;19:536-43.

40. Flores-Barrera E, Thomases DR, Cass DK, Bhandari A, Schwarcz R, Bruno JP, et al. Preferential Disruption of Prefrontal GABAergic Function by Nanomolar Concentrations of the alpha7nACh Negative Modulator Kynurenic Acid. J Neurosci. 2017;37:7921-29.

41. Zhang W, Rosenkranz JA. Repeated restraint stress enhances cue-elicited conditioned freezing and impairs acquisition of extinction in an age-dependent manner. Behav Brain Res. 2013;248:12-24.

42. Wohr M, Orduz D, Gregory P, Moreno H, Khan U, Vorckel KJ, et al. Lack of parvalbumin in mice leads to behavioral deficits relevant to all human autism core symptoms and related neural morphofunctional abnormalities. Transl Psychiatry 2015;5:e525.

43. Caballero A, Thomases DR, Flores-Barrera E, Cass DK, Tseng KY. Emergence of GABAergic-dependent regulation of input-specific plasticity in the adult rat prefrontal cortex during adolescence. Psychopharmacol (Berl). 2014; 231:1789-96.

44. Floresco SB, Seamans JK, Phillips AG. Selective roles for hippocampal, prefrontal cortical, and ventral striatal circuits in radial-arm maze tasks with or without a delay. J Neurosci. 1997;17:1880-90.

45. Chudasama Y, Doobay VM, Liu Y. Hippocampal-prefrontal cortical circuit mediates inhibitory response control in the rat. J Neurosci. 2012;32:10915-24.

46. Gilmartin MR, Kwapis JL, Helmstetter FJ. Trace and contextual fear conditioning are impaired following unilateral microinjection of muscimol in the ventral hippocampus or amygdala, but not the medial prefrontal cortex. Neurobiol Learn Mem. 2012;97:452-64.

47. Sierra-Mercado D, Padilla-Coreano N, Quirk GJ. Dissociable roles of prelimbic and infralimbic cortices, ventral hippocampus, and basolateral amygdala in the expression and extinction of conditioned fear. Neuropsychopharmacology 2011;36:529-38.

48. Belforte JE, Zsiros V, Sklar ER, Jiang Z, Yu G, Li Y, et al. Postnatal NMDA receptor ablation in corticolimbic interneurons confers schizophrenia-like phenotypes. Nat Neurosci. 2010;13:76-83.

49. Brown JA, Ramikie TS, Schmidt MJ, Baldi R, Garbett K, Everheart MG, et al. Inhibition of parvalbumin-expressing interneurons results in complex behavioral changes. Mol Psychiatry 2015;20:1499-507.

50. Carlen M, Meletis K, Siegle JH, Cardin JA, Futai K, Vierling-Claassen D, et al. A critical role for NMDA receptors in parvalbumin interneurons for gamma rhythm induction and behavior. Mol Psychiatry 2012;17:537-48.

51. Cho KK, Hoch R, Lee AT, Patel T, Rubenstein JL, Sohal VS. Gamma rhythms link prefrontal interneuron dysfunction with cognitive inflexibility in Dlx5/6(+/-) mice. Neuron 2015;85:1332-43.

52. Lazarus MS, Krishnan K, Huang ZJ. GAD67 deficiency in parvalbumin interneurons produces deficits in inhibitory transmission and network disinhibition in mouse prefrontal cortex. Cereb Cortex 2015;25:1290-6. 
53. Murray AJ, Woloszynowska-Fraser MU, Ansel-Bollepalli L, Cole KL, Foggetti A, Crouch $\mathrm{B}$, et al. Parvalbumin-positive interneurons of the prefrontal cortex support working memory and cognitive flexibility. Sci Rep. 2015;5:16778.

54. de Lecea $L$, del Rio JA, Soriano E. Developmental expression of parvalbumin mRNA in the cerebral cortex and hippocampus of the rat. Brain Res Mol Brain Res. 1995;32:1-13.

55. Hof PR, Glezer II, Conde F, Flagg RA, Rubin MB, Nimchinsky EA, et al. Cellular distribution of the calcium-binding proteins parvalbumin, calbindin, and calretinin in the neocortex of mammals: phylogenetic and developmental patterns. J Chem Neuroanat. 1999;16:77-116.

56. Vogt Weisenhorn DM, Celio MR, Rickmann M. The onset of parvalbuminexpression in interneurons of the rat parietal cortex depends upon extrinsic factor(s). Eur J Neurosci. 1998;10:1027-36.

57. Lucas EK, Dougherty SE, McMeekin LJ, Reid CS, Dobrunz LE, West AB, et al. PGC1alpha provides a transcriptional framework for synchronous neurotransmitter release from parvalbumin-positive interneurons. J Neurosci. 2014;34:14375-87.

58. Lucas EK, Markwardt SJ, Gupta S, Meador-Woodruff JH, Lin JD, OverstreetWadiche $L$, et al. Parvalbumin deficiency and GABAergic dysfunction in mice lacking PGC-1alpha. J Neurosci. 2010;30:7227-35.

59. Lew SE, Tseng KY. Dopamine modulation of GABAergic function enables network stability and input selectivity for sustaining working memory in a computational model of the prefrontal cortex. Neuropsychopharmacology 2014;39:3067-76.

60. Caillard O, Moreno H, Schwaller B, Llano I, Celio MR, Marty A. Role of the calciumbinding protein parvalbumin in short-term synaptic plasticity. Proc Natl Acad Sci USA. 2000;97:13372-7.

61. Vreugdenhil M, Jefferys JG, Celio MR, Schwaller B. Parvalbumin-deficiency facilitates repetitive IPSCs and gamma oscillations in the hippocampus. J Neurophysiol. 2003;89:1414-22.

62. Gabbott P, Headlam A, Busby S. Morphological evidence that CA1 hippocampal afferents monosynaptically innervate PV-containing neurons and $\mathrm{NADPH}$ diaphorase reactive cells in the medial prefrontal cortex (Areas 25/32) of the rat. Brain Res. 2002;946:314-22.
63. Gabbott PL, Warner TA, Busby SJ. Amygdala input monosynaptically innervates parvalbumin immunoreactive local circuit neurons in rat medial prefrontal cortex. Neuroscience 2006;139:1039-48.

64. Benes FM. Myelination of cortical-hippocampal relays during late adolescence. Schizophr Bull 1989;15:585-93.

65. Caballero A, Diah KC, Tseng KY. Region-specific upregulation of parvalbumin-, but not calretinin-positive cells in the ventral hippocampus during adolescence. Hippocampus 2013;23:1331-6.

66. Orduz D, Bischop DP, Schwaller B, Schiffmann SN, Gall D. Parvalbumin tunes spike-timing and efferent short-term plasticity in striatal fast spiking interneurons. J Physiol. 2013;591:3215-32.

67. Thomson AM. Facilitation, augmentation and potentiation at central synapses. Trends Neurosci. 2000;23:305-12.

68. Holt DJ, Lebron-Milad K, Milad MR, Rauch SL, Pitman RK, Orr SP, et al. Extinction memory is impaired in schizophrenia. Biol Psychiatry 2009;65:455-63.

69. Uliana DL, Resstel LBM, Grace AA. Fear extinction disruption in a developmental rodent model of schizophrenia correlates with an impairment in basolateral amygdala-medial prefrontal cortex plasticity. Neuropsychopharmacology 2018;43:2459-67.

70. McMeekin LJ, Lucas EK, Meador-Woodruff JH, McCullumsmith RE, Hendrickson RC, Gamble KL, et al. Cortical PGC-1alpha-Dependent Transcripts Are Reduced in Postmortem Tissue From Patients With Schizophrenia. Schizophr Bull 2016;42:1009-17.

71. Cabungcal JH, Counotte DS, Lewis E, Tejeda HA, Piantadosi P, Pollock C, et al. Juvenile antioxidant treatment prevents adult deficits in a developmental model of schizophrenia. Neuron 2014;83:1073-84.

72. Steullet P, Cabungcal JH, Coyle J, Didriksen M, Gill K, Grace AA, et al. Oxidative stress-driven parvalbumin interneuron impairment as a common mechanism in models of schizophrenia. Mol Psychiatry 2017;22:936-43.

73. Eggermann $E$, Jonas P. How the 'slow' $\mathrm{Ca}(2+)$ buffer parvalbumin affects transmitter release in nanodomain-coupling regimes. Nat Neurosci. 2011;15:20-2. 